\title{
TERT inhibited the ER stress-induced cell death in normal, neuronal and cancerous cells
}

\author{
Amer A. Abd El-Hafeez ${ }^{1,2,3}$, Toru Hosoi ${ }^{1}$, Kanako Nakatsu ${ }^{1}$, Akira Shimamoto ${ }^{4}$, Hidetoshi Tahara ${ }^{4}$, \\ Koichiro Ozawa ${ }^{1}$
}

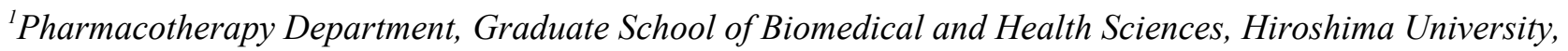
Hiroshima, Japan, ${ }^{2}$ Global Career Design Center, Hiroshima University, Hiroshima, Japan, ${ }^{3}$ Pharmacology and Experimental Oncology Unit, Cancer Biology Department, National Cancer Institute, Cairo University, Cairo, Egypt, ${ }^{4}$ Department of Cellular and Molecular Biology, Graduate School of Biomedical and Health Sciences, Hiroshima University, Hiroshima, Japan

Background: The implication of the endoplasmic reticulum (ER) stress has been shown in many diseases including aging, neurodegenerative diseases, and cancer. Therefore, strategies that attenuate the ER stress in these diseases would be beneficial for their treatment.

Materials and methods: RT-PCR was used to test the expression of hTERT, GRP78, c-myc, p23 or GAPDH mRNA. Retroviral method was used to transduce TERT in in three different human cell lines; normal (TIG-3), neuronal (SHSY5Y), and Cancerous (MCF-7) cells. RT-PCR was used to confirm that TERT was over expressed. The viability of cells was estimated by the lactate dehydrogenase (LDH) leakage method as well as the crystal violet assay.

Results: To explore how TERT stand against the stress, we investigated the possible link between ER stress and telomerase. We observed an increase in GRP78 mRNA levels on tunicamycin-treatment, inducing ER stress. Thus, we next analyzed levels of TERT, p23 and c-myc in ER stressed cells. We found that TERT levels increased $2 \mathrm{~h}$ after tunicamycin-treatment, which decreased thereafter. There was no increase in c-myc or p23 levels in the tunicamycin (12 h)-treated cells. Furthermore, we observed that TERT inhibited the ER stress-induced cell death in normal, neuronal and cancerous cells. Furthermore, we found that TERT is able to inhibit the ER stress-induced cell death and enhance the cell survival regardless of the ER stress inducer, including tunicamycin (protein glycosylation inhibitor), thapsigargin $\left(\mathrm{Ca}^{2+}-\right.$ ATPase inhibitor), brefeldin A (protein transport inhibitor), or dithiothreitol (DTT; disulfide-bond formation inhibitor). The effect may be mediated through TERT enzyme activity because telomerase specific inhibitor, BIBR1532, attenuated these responses.

Conclusion: Collectively, these data may suggest the ability of TERT to suppress the ER stress. These data may offer new insights regarding the implication of TERT in the treatment of ER stress-induced diseases such as aging, cancer, and neurodegenerative diseases.

Keywords: Telomerase reverse transcriptase, ER stress, cell death 